\title{
INICIO DE LA ALFABETIZACIÓN, HABILIDADES PRELECTORAS Y CONTEXTO ALFABETIZADOR FAMILIAR EN UNA MUESTRA DE NIÑOS URUGUAYOS
}

\section{LITERACY ACQUISITION, PRE-READING SKILLS AND FAMILY LITERACY CONTEXT IN A SAMPLE OF URUGUAYAN CHILDREN}

\author{
Ariel Cuadro \\ Juana Berná
}

\author{
Universidad Católica del Uruguay
}

\begin{abstract}
Resumen: Los efectos de las habilidades prelectoras así como del contexto familiar alfabetizador en el inicio de la alfabetización, tienen implicaciones significativas tanto para los avances teóricos como para las prácticas educativas relacionadas con la adquisición de la habilidad lectora. El objetivo de este estudio fue analizar las relaciones entre el desarrollo de las habilidades prelectoras, entendidas en términos de alfabetización emergente, el contexto alfabetizador familiar y el inicio de la alfabetización, a partir de una muestra de 56 niños de educación inicial. Se aplicaron pruebas para evaluar el conocimiento léxico, habilidades metafonológicas, velocidad de nominación y reconocimiento de letras, así como un cuestionario para conocer el contexto alfabetizador familiar de los niños participantes. Los resultados dan muestra de una relación significativa entre las habilidades prelectoras y el inicio de la alfabetización, así como de la importancia de las prácticas alfabetizadoras familiares.
\end{abstract}

Palabras Clave: lectura; habilidades prelectoras; contexto alfabetizador familiar; alfabetización temprana

\begin{abstract}
The effects of pre-reading skills and family context at the beginning of literacy have significant implications for both theoretical advances and for educational practices related to the acquisition of literacy. The aim of this study was to analyze the relationship between the development of pre-reading skills, understood in terms of emergent literacy, family literacy context and the beginning of literacy in a sample of 56 preschool children. To assess lexical knowledge, metaphonological skills, naming speed and letter recognition, different tests were used, as well as a questionnaire to study the family literacy context of the participating children. The results show a significant relationship between pre-reading skills and the beginning of literacy acquisition as well as the importance of family literacy practices.
\end{abstract}

Key Words: reading; pre-reading skills; family literacy context; early literacy

\section{Introducción}

La lectura es una actividad cultural que permite la transmisión de conocimientos a través de prácticas sociales y comunicativas. Pero también es una actividad cognitiva compleja en la que intervienen numerosos procesos mentales de manera totalmente sincronizada (Cuetos, Rodríguez, Ruano \& Arribas, 2007; Defior, 2008; Morais, 1998), algunos específicos de la lectura y otros más generales, pero no por ello menos importantes, como son los proceso motivacionales y afectivos (Serrano, 2005).

Desde el paradigma de alfabetización emergente 0 emergent literacy (Whitehurst y Lonigan, 1998; Bravo-Valdivieso, 2002; Guardia, 2003; Dickinson y Newman, 2006) o más específicamente desde los estudios de la psicolingüística cognitiva (Alegría, 2006), variables como el conocimiento léxico (vocabulario), la velocidad de nominación, las habilidades metafonológicas y el conocimiento del principio alfabético constituyen componentes esenciales en el proceso de adquisición del lenguaje escrito (Bravo, 1993; Wimmer, Mayringer y Landerl, 2000; Cuadro, 2010) en tanto que permiten el desarrollo de los mecanismos de reconocimiento de palabras y la automatización de los mismos (acceso léxico). De esta forma se automatizan los procesos que implican asociar la forma ortográfica (secuencias de grafemas) de cada palabra con su forma fonológica.

Dichos procesos cognitivos interaccionan con factores ambientales del contexto lector, 
como lo son el sistema ortográfico de una determinada lengua en la que se aprende a leer, los materiales de enseñanza que se emplean, la importancia concedida a ésta y el ambiente o contexto familiar (Serrano y Defior, 2004).

Las variables contextuales (familiares, escolares y sociales) inciden en el desarrollo de la lectura, en la actitud que se desarrolla hacia ella (Andrés, Canet-Juric, Introzzi \& Urquijo, 2010) y en el uso que se hace de ella, así como en otras variables motivacionales y afectivas que están implicadas en todo proceso cognitivo (Beltrán \& Téllez, 2002).

Un ambiente alfabetizador familiar adecuado favorece el desarrollo de las habilidades verbales, enriquece el vocabulario y aumenta el interés por la lectura (Alegría \& Corderó, 2000). Los padres y el entorno familiar del niño ocupan un lugar de suma importancia en la preparación de la lectoescritura (Herrera, 2002; Samuelson \& Lundberg, 2003; Herrera, Defior \& Lorenzo, 2007; Andrés, Urquijo, Navarro \& García-Sedeño, 2010) y en el desarrollo de la lectura. Así, por ejemplo lo niños que además de estar escolarizados reciben en sus hogares estímulos para leer, muestran un mejor rendimiento lector que aquellos que no lo están (Stanovich, 1988; Cunninhan \& Stanovich, 1990).

Mientras que para la adquisición del lenguaje oral únicamente es necesario contar con un entorno de estimulación natural del lenguaje, en la adquisición de la lectura resulta imprescindible la presencia de una instrucción formal, sistemática e intencional (Selles-Nohales \& Martínez-Giménez, 2008; Alegría, Carrillo \& Calvo, 2001). La lectura se adquiere y se consolida gracias a la interacción de las habilidades cognitivas explicitadas anteriormente con la instrucción intencional y la estimulación que proporciona el ambiente familiar (Selles Nohales \& Martínez Giménez, 2008; Jiménez \& O'Shanahan, 2008). Asimismo cuando un niño inicia el aprendizaje formal de la lectura, cuenta con un bagaje de habilidades fonológicas, semánticas y léxicas (Serrano, 2005), procedentes del lenguaje oral, que influyen en su desarrollo lector y que a la vez son retroalimentadas por la adquisición del lenguaje escrito.

Andrés et al. (2010) utilizan el concepto de contexto alfabetizador al referirse al conjunto de recursos y experiencias hogareñas relativas al contacto con la lectura y la escritura, en las que participa el niño durante sus años de edu- cación preescolar. Estos autores al evaluar el contexto alfabetizador familiar de una muestra de 52 niños argentinos de 5 años, encontraron relaciones significativas entre el nivel educativo de los padres y la adquisición de habilidades prelectoras de los niños, consideradas en términos de conocimiento del material gráfico y de la conciencia lingüística (Andrés et al., 2010). Trabajos similares, realizados en poblaciones carenciadas, encontraron correlaciones entre diferentes variables del contexto alfabetizador; así aparecieron correlaciones moderadas entre las prácticas alfabetizadoras paternas y algunas características infantiles informadas por los padres en torno a experiencias de lectura (Querejeta, 2010). Piacente, Marder, Resches y Ledesma (2006) al comparar las características del contexto alfabetizador hogareño en familias en situación de pobreza y de familias de sectores socioeconómicos promedio, encontraron diferencias significativas entre las variables del contexto alfabetizador dependiendo del grupo.

El estudio de las variables prelectoras y en particular el contexto alfabetizador constituyen una buena herramienta para predecir futuras dificultades en el aprendizaje lector. Por tanto, la constatación de factores de riesgo en el aprendizaje de la lectura, antes de que comience la enseñanza formal, permite afrontar el problema desde una perspectiva preventiva y abordar una remediación más temprana (Alegría, 2010).

Los efectos de las habilidades prelectoras así como del contexto familiar alfabetizador en el inicio de la alfabetización, tienen implicaciones significativas para el desarrollo teórico y para las prácticas educativas relacionadas con la adquisición de la lectura. El objetivo de este trabajo es contribuir al conocimiento de estas relaciones, aportando nuevos datos empíricos sobre el inicio de la alfabetización, el desarrollo de las habilidades prelectoras, entendida en términos de alfabetización emergente y el contexto alfabetizador familiar.

\section{Método}

\section{Participantes}

La muestra estuvo integrada por 56 niños y niñas de educación inicial de entre 4 y 5 años de edad, de un colegio privado de nivel socioeconómico medio de la ciudad de 
Colonia. Todos los participantes eran hispanohablantes, 24 eran niñas y 32 varones y los informantes del protocolo del Contexto Alfabetizador Familiar fueron representados por 53 madres y 3 padres.

\section{Instrumentos}

Contexto alfabetizador familiar. Se utilizó el protocolo autoadministrable de opción múltiple denominado "Evaluación del Contexto Alfabetizador Familiar" (Piacente, Querejeta, Marder \& Resches, 2003), que posee 72 preguntas de múltiple opción y explora las siguientes áreas: Identificación del parentesco del informante con el niño, características del hogar, características del niño, características de los padres y hermanos y creencias y prácticas alfabetizadoras.

En este estudio se emplean 36 preguntas del protocolo original que se focalizan en el contexto alfabetizador, quedando 20 de ellas categorizadas en las siguientes áreas: 1. Disponibilidad de material que favorece la alfabetización. 2. Tiempo de lectura diaria de los padres 3 . Motivación y producción infantil. 4. Prácticas alfabetizadoras. Los resultados se clasifican en tres niveles: favorecedor, intermedio y poco favorecedor.

Las restantes 16 preguntas identifican la lengua hablada en el hogar, la atribución de la responsabilidad de la enseñanza de la lectura a padres o maestros, los estudios realizados por los padres, disfrute de la actividad lectora y las visitas realizadas a bibliotecas.

\section{Evaluación de las habilidades prelectoras}

- Evaluación del vocabulario. Se utilizó el Test de vocabulario en imágenes Peabody (Dunn, Padilla \& Lugo, 1986) que evalúa el nivel de vocabulario receptivo del sujeto y cuya fiabilidad es de $\alpha=.91$. La prueba consta de 125 ítems organizados en orden de dificultad en donde se le pide al niño que señale en una lámina, que se le presenta, la imagen de la palabras que el examinador le indica verbalmente.

- Velocidad de Nominación. Se utilizó la prueba Rapid Automatized Naming Tests (RAN) (Wolf \& Denckla, 2003). Esta técnica evalúa la velocidad de nominación de letras, números, colores y objetos y se indica una fiabilidad de $\alpha=.81$. La prueba consta de cua- tro tareas: serie de letras, serie de números, serie de colores y serie de objetos. Se le pide al sujeto, que nombre lo más rápido posible los estímulos de cada fila de izquierda a derecha y se contabiliza el tiempo de ejecución.

- Prueba de evaluación del Conocimiento Fonológico. Se utilizó la herramienta PSEFA (González, 1993), que consta de una prueba de identificación de sonidos formada por 13 pares de palabras, donde se debe identificar el sonido propuesto, y una prueba de omisión de sonidos de 6 pares de palabras donde se debe omitir el fonema inicial. Se puntúan los aciertos.

- Evaluación de iniciación de la alfabetización. Se utilizó el subtest Identificación de letras de la prueba PROLEC-R (Cuetos et al., 2007), en la que el niño debe identificar el nombre o sonido de 20 letras, que se le presentan. A los niños de 4 años se les presentaron las 20 letras en mayúscula. La fiabilidad es de $\alpha=.49$.

\section{Procedimiento}

Una vez obtenida la autorización de la gerencia del colegio para realizar la investigación, se procedió a evaluar a los participantes de forma individual, en dos sesiones de 40 minutos cada una durante el horario escolar, en una sala facilitada por el centro educativo para este fin. En la primera sesión se aplicaron las pruebas de evaluación del vocabulario y el subtest de Identificación de letras de la prueba PROLEC-R. En la segunda se hicieron las evaluaciones de velocidad de nominación y conocimiento fonológico.

El protocolo del Contexto Alfabetizador Familiar, junto con una carta de presentación, se entregó a los padres por medio del centro escolar, que debían devolverlo al docente del colegio, al finalizar.

\section{Resultados}

En relación al nivel de estudios de los padres, los resultados reflejan un promedio de 13,47 años de escolarización de las madres y de 12,79 años de los padres, siendo la media de ambos 13,1 y señalando, que tres madres $y$ un padre no fueron escolarizados y seis no contestaron.

Con respecto al disfrute de la actividad lectora, el $73 \%$ de las madres informaron un nivel alto de disfrute de la lectura, frente a un $43 \%$ de los padres, un $18 \%$ de las madres 
señalaron un nivel bajo de disfrute de la lectura, frente a un $35 \%$ de los padres y un $4 \%$ no disfrutaban en absoluto con la lectura al igual que un $7 \%$ de los padres. El $3 \%$ de las madres no contestaron.

Asimismo el $89 \%$ de los informantes piensan que los responsables de la enseñanza de la lectura son los padres y maestros, mientras que el $4 \%$ opina, que la responsabilidad recae solo en los padres y un $6 \%$ se la adjudica a los maestros ( $1 \%$ no contestó). El $45 \%$ de los padres no realizan visitas a bibliotecas con sus hijos, mientras el $41 \%$ cumple con esta rutina 1 o 2 veces al mes, el 5\% 1 o 2 veces por semana y el $4 \%$ asiduamente (el $5 \%$ de los informantes no contestaron esta pregunta).

En la Tabla 1 se reflejan los puntajes promedio y la desviación estándar de las variables del contexto alfabetizador, en las cuales se vislumbra que la mayoría de los hogares se encuentra dentro del rango favorecedor para la alfabetización en relación a la disponibilidad de material, tiempo de lectura de los padres, prácticas alfabetizadoras y motivación y producción del niño.

En la Tabla 2 se presentan las correlaciones entre las variables del contexto alfabetizador analizadas: disponibilidad del material de lectura, hábito de lectura de los padres, prácticas alfabetizadoras y motivación y producción del niño.

En la Tabla 3 se muestran las medias y las desviaciones estándar de los resultados de las pruebas de habilidades prelectoras (vocabulario, velocidad de nominación, identificación de sonido inicial, omisión de sonido inicial),

\begin{tabular}{|c|c|}
\hline $\begin{array}{l}\text { Variables del contexto } \\
\text { alfabetizador }\end{array}$ & $\begin{array}{l}\text { Puntaje } \\
\text { promedio (DS) }\end{array}$ \\
\hline Disponibilidad del material & $8.36(0.99)$ \\
\hline Hábitos lectores de los padres & $3.31(1.25)$ \\
\hline Prácticas alfabetizadoras & $18.91(2.25)$ \\
\hline $\begin{array}{l}\text { Motivación y producción del } \\
\text { niño }\end{array}$ & $22.37(2.00)$ \\
\hline
\end{tabular}

Tabla 2

Correlaciones entre las variables de contexto alfabetizador

\begin{tabular}{lcccc}
\hline & $\begin{array}{c}\text { Disponibilidad } \\
\text { del material }\end{array}$ & $\begin{array}{c}\text { Hábitos de } \\
\text { lectura de los } \\
\text { padres }\end{array}$ & $\begin{array}{c}\text { Prácticas } \\
\text { alfabetizadoras }\end{array}$ & $\begin{array}{c}\text { Motivación y } \\
\text { producción del } \\
\text { niño }\end{array}$ \\
\hline Disponibilidad del material & 1 & $.36^{* *}$ & .07 & .16 \\
Hábitos de lectura de los padres & $.36^{* *}$ & 1 & .06 & .07 \\
Prácticas alfabetizadoras & .71 & .06 & 1 & $.60^{* *}$ \\
Motivación y producción del niño & .16 & .07 & $.60^{* *}$ & 1 \\
\hline
\end{tabular}

Tabla 3

Puntajes promedios y desviación estándar en las variables de

habilidades prelectoras y reconocimiento de letras

\begin{tabular}{ll}
\hline Habilidades prelectoras & $\begin{array}{l}\text { Media y desviación } \\
\text { estándar }\end{array}$ \\
\hline Omisión sonido inicial & $8.74(3.70)$ \\
Identificación de sonidos & $1.69(2.48)$ \\
Vocabulario & $35.11(13.32)$ \\
Velocidad de nominación & $300.2(99.64)$ \\
Reconocimiento de letras & $11.84(6.47)$ \\
\hline
\end{tabular}


así como de la medida de reconocimiento de letras, mientras en la Tabla 4 se presentan los análisis de las relaciones entre dichos resultados.

Como las variables elegidas no cumplen con los criterios necesarios para aplicar pruebas paramétricas, medido en términos de homocedasticidad (estadístico de Levene: $\mathrm{p}<.05$ ) y normalidad (Prueba de KolmogorovSmirnov: $p<.05$ ) de los datos, se analizaron con estadística no paramétrica las correlaciones entre las variables del contexto alfabetizador y las habilidades prelectoras y el reconocimiento de letras (Tabla 5).

Por último, con el fin de poder evaluar el grado en que las variables del contexto alfabetizador podrían incidir en el inicio a la alfabetización de los niños participantes, medida a través del nivel de reconocimiento de letras, se utilizó el análisis discriminante. Se estableció un criterio basado en centiles para diferenciar dos niveles de inicio a la alfabetización a partir de la prueba de reconocimiento de letras: un nivel bajo correspondiente a puntajes por debajo del percentil 30 y un nivel alto equivalente a resultados superiores a esta medida. Cada grupo se clasificó de acuerdo a las variables definidas como contexto alfabetizador: disponibilidad de materiales, hábitos de lectura de los padres, prácticas alfabetizadora, y motivación y producción de los niños. Se utilizó el método de selección de pasos con el fin de eliminar las variables que no contribuían a mejorar la clasificación, dejando eliminadas las medidas de Hábitos de lectura de los padres ( $F, 0,164)$; Disponibilidad de materiales ( $F, 0,015)$; Motivación y producción ( $F, 8,081)$, por presentar una alta colinealidad con las restantes variables. En la Tabla 6 se presentan las variables seleccionadas.

Tabla 4

Correlaciones entre las variables de las habilidades prelectoras y reconocimiento de letras

\begin{tabular}{lccccc}
\hline & $\begin{array}{c}\text { Omisión } \\
\text { sonido inicial }\end{array}$ & $\begin{array}{c}\text { Identificación } \\
\text { sonidos }\end{array}$ & $\begin{array}{c}\text { Velocidad } \\
\text { de } \\
\text { nominación }\end{array}$ & Vocabulario & $\begin{array}{c}\text { Reconocimiento } \\
\text { de letras }\end{array}$ \\
\hline Omisión sonido inicial & 1 & $.57^{* *}$ & $.60^{* *}$ & .23 & $.52^{* *}$ \\
Identificación sonidos & $.57^{* *}$ & 1 & $-.37^{*}$ & $.46^{* *}$ & $.47^{* *}$ \\
Velocidad de nominación & $-.61^{* *}$ & $-.37^{* *}$ & 1 & -.28 & $-.60^{* *}$ \\
Vocabulario & $.35^{* *}$ & $.46^{* *}$ & -.28 & 1 & .23 \\
Reconocimiento de letras & $.52^{* *}$ & $.47^{* *}$ & $-.60^{* *}$ & .23 & 1 \\
\hline
\end{tabular}

Tabla 5

Resultados (Chi-cuadrado) de la prueba Kruskal-Wallis para las comparaciones entre las variables de contexto alfabetizador y las de habilidades prelectoras y reconocimiento de letras

\begin{tabular}{lccccc}
\hline & $\begin{array}{c}\text { Omisión } \\
\text { sonido inicial }\end{array}$ & $\begin{array}{c}\text { Identificación } \\
\text { sonidos }\end{array}$ & $\begin{array}{c}\text { Velocidad } \\
\text { de } \\
\text { nominación }\end{array}$ & Vocabulario & $\begin{array}{c}\text { Reconocimiento } \\
\text { de letras }\end{array}$ \\
\hline Disponibilidad del material & .42 & 1.87 & .64 & 1.19 & .13 \\
Hábitos de lectura padres & .23 & .62 & 3.90 & 1.20 & .53 \\
$\begin{array}{l}\text { Prácticas alfabetizadoras } \\
\text { Motivación y producción del }\end{array}$ & .77 & .08 & 1.31 & 1.07 & 1.76 \\
niño & .19 & .09 & .25 & .45 & 1.54 \\
\hline
\end{tabular}

Tabla 6

Variable seleccionada en el análisis discriminante siguiendo el

análisis de selección por pasos

\begin{tabular}{lcc}
\hline Introducidas & Lambda de Wilks & $\mathrm{P}$ \\
\hline Prácticas alfabetizadoras & 0,464 & 0,004 \\
\hline
\end{tabular}


Los resultados de la clasificación final aparecen en la Tabla 7, en la cual se muestra que el $75 \%$ de los niños fueron clasificados exactamente en sus propios niveles de inicio de la alfabetización.

Tabla 7

Resultados de la clasificación terminado el análisis discriminante

\begin{tabular}{lccc}
\hline $\begin{array}{l}\text { Reconocimiento } \\
\text { de letras }\end{array}$ & \multicolumn{2}{c}{$\begin{array}{c}\text { Pertenencia a grupos } \\
\text { pronosticada }\end{array}$} & Total \\
\hline \multirow{4}{*}{ Alto } & Bajo & \\
Alto & 35 & 3 & 38 \\
Bajo & 11 & 7 & 18 \\
\hline
\end{tabular}

\section{Discusión}

El presente estudio se propuso analizar las relaciones entre las habilidades prelectoras, el inicio de la alfabetización y el contexto familiar alfabetizador en una muestra de niños de educación inicial.

El análisis de las relaciones entre las variables prelectoras y con el reconocimiento de letras, que da muestra del inicio de la alfabetización, se comportan en el marco de los hallazgos de las investigaciones en psicología de la lectura. Los resultados en las pruebas que evalúan las habilidades metafonológicas correlacionan significativamente entre sí (.57) y con el reconocimiento de letras (.52 y .47), al igual que esta última con la velocidad de nominación (-.60). El reconocimiento de las letras indica el grado de conocimiento del principio alfabético, el que debe ser adquirido por los lectores principiantes para poder desarrollar los mecanismos de acceso léxico, lo que supone el desarrollo temprano de habilidades metafonológicas (Castells, 2009). Asimismo la velocidad de nominación, entendida como la capacidad de recuperación de códigos fonológicos desde la memoria a largo plazo para la pronunciación de letras, dígitos y palabras (De Jong \& Van Der Leij, 1999), se vincula con la fluidez, velocidad y exactitud en la identificación de las palabras (Wimmer et al., 2000; Wolf \& Bowers, 2000). La precisión y la velocidad con las que se identifican las palabras, se traduce en la automatización del acceso lector (Perfetti, 1992; Cuadro, 2010), permitiendo la liberación de recursos cognitivos, para aumentar la eficacia de los procesos cognitivos superiores implicados en la comprensión (Lesgold, Resnick \& Hammond, 1985; Perfetti, 1986; Britton \& Graesser, 2014).

En el análisis del contexto familiar alfabetizador en general no aparecen diferencias significativas entre las variables consideradas. Este resultado se considera previsible, ya que los participantes eran niños pertenecientes a la misma institución educativa y de una misma localidad pequeña del interior del país. En su mayoría se trataba de hogares favorecedores de la alfabetización, mostrando una mayor diferencia en relación a dos prácticas alfabetizadoras: la edad de inicio de la lectura y la frecuencia de la lectura. A igual que en el estudio de Piacente et al. (2006) llama la atención la diferencia encontrada entre las prácticas alfabetizadoras y las producciones infantiles, sobre las que informan los padres. Este dato puede interpretarse como un sesgo de la deseabilidad social por parte de los padres.

Se observa un peso relativo mayor entre las prácticas alfabetizadoras y la motivación y producciones de los niños, coincidente con otros trabajos (Piacente et al., 2006; Burgues, Hecht \& Loningan, 2002), pero no se encuentran relaciones estadísticamente significativas entre las variables de contexto alfabetizador y las habilidades prelectoras, ni con la medida de reconocimiento de letras. Andrés et al. (2010) en un estudio similar identificaron que en los hogares, en los que los padres tienen mayores niveles de escolarización, se observaron mejores niveles de desempeño en habilidades prelectoras. Este dato se atribuye a que en estos hogares se ofrecen mayor cantidad de recursos y experiencias vinculadas a situaciones de lectura y escritura.

Las prácticas alfabetizadoras correlacionan positivamente y significativamente con la motivación y producción del niño (.60), de tal forma que la familia resulta un buen aliado en la estimulación por el gusto en la lectura. La motivación infantil ha sido objeto de especial interés tanto en lo que se refiere al desarrollo intrínseco del deleite lector como en la planificación de actividades que la dinamicen extrínsecamente. Cuando un niño está motivado, desde un punto de vista pedagógico, ya supone un estimulo positivo para su alfabetización (Quintanal, 2001).

Si se analizan los niveles de inicio de la alfabetización, en niños en una misma situación de escolarización, se observa que las prácticas alfabetizadoras muestran una buena discrimi- 
nación de estos niveles. En el presente estudio, el análisis discriminante realizado, diferenciando niveles de inicio de la alfabetización a partir de la lectura de letras, indica que la función discriminante para caracterizar los niveles de inicio a la alfabetización es precisamente la variable prácticas alfabetizadoras. Como se ha señalado, diversos estudios muestran la importancia de las interacciones entre los padres y los hijos en momentos de lectura compartida, antes de que los niños inicien su aprendizaje formal del lenguaje escrito (Goddard, Durkin \& Rutter, 1985; Jiménez \& O'Shanahan, 2008).

Implicancias educativas se desprenden de este estudio al ratificar la importancia de las intervenciones alfabetizadoras tempranas en la educación infantil, que atiendan a las diferencias que se puedan dar en los hogares, en particular en cuanto a las prácticas alfabetizadoras. A su vez resulta relevante profundizar en el trabajo con los padres para que promuevan contextos alfabetizadores favorables.

\section{Referencias}

Alegría C., \& Corderó, C. (2000). La familia, instrumento de prevención en la lectoescritura. Valencia: Congreso Mundial de Lecto-escritura.

Alegría, J., Carrillo, M., \& Calvo, A. (2001). El inicio del aprendizaje de la lectura en educación infantil. Madrid: Santillana Servicios educativos.

Alegría, J. (2006). Por un enfoque psicolingüístico del aprendizaje de la lectura y sus dificultades-20 años después-. Infancia y Aprendizaje, 29, 93-111.

Alegría, J. (2010). Informe sobre las "buenas prácticas" relativas a la dislexia en los países de lengua española. Foro Mundial de la Dislexia. UNESCO. Paris, 3-5 febrero-2010.

Andrés, M.L., Canet -Juric, R., Introzzi, I., \& Urquijo, S. (2010). Disponibilidad de recursos materiales en el hogar y adquisición de habilidades prelectoras. Revista semestral de Associacao Brasileira de Psicologia Escolar e Educacional, SP. 14 (1),139148.

Andrés, M.L., Urquijo, S., Navarro, J., \& García-Sedeño, M. (2010). Contexto alfabetizador familiar: relaciones con la adquisición de habilidades prelectoras y desempeño lector. European Journal of Education and Psychology 3 (1), 129-140.

Beltrán , S., \& Téllez, J. (2002). El papel de la escuela y la familia en la animación y el aprendizaje de la lectura. Facultad de educación de la UNED. Madrid: Facultad de Educacion UNED. Recuperado de http://www.researchgate.net/profile/Jose_Tellez2/ publication/264556525_El_papel_de_la_escuela_y_ la_familia_en_la_animacin_y_el_aprendizaje_de_ la lectura/links/53e75d340cf2fb748721999e.pdf

Bravo Valdivieso, L. (2002). La conciencia fonológica como una zona de desarrollo próximo para el aprendizaje inicial de la lectura. Estudios Pedagógicos, 28,165-177.
Bravo, L. (1993). La Dislexia: cien años después. Investigación, Antecedentes históricos y definiciones. Psyké, 2, 95-105.

Britton, B.; Graesser, A. (2014). Models of Understanding. NY: Psychology Press.

Burgues, S.R., Hecht, S.A., \& Loningan, C.J., (2002). Relations of the home literaracy environment (HLE) to the development of reading-related abilities: a oneyear longitudinal study, Reading Research Quarterly, 37(4), 408-426.

Castells, N. (2009). La investigación sobre la enseñanza y aprendizaje de la lectura inicial: revisión y clasificación. Infancia y Aprendizaje, 32 (1), 33-48.

Cuadro, A. (2010). La lectura y sus dificultades. Dislexia Evolutiva. Montevideo: Grupo Magró.

Cuetos, F., Rodríguez, B., Ruano, E., \& Arribas, D. (2007). PROLEC-R. Madrid: Tea ediciones.

Cunninhan, A.C. \& Stanovich, K.E. (1990). Assessing print exposure and orthographic processing in children: A quick measure of reading experience. Journald of Education Psychology, 28, 733-740.

De Jong, P. \& Van der Leij, A. (1999). Specific contributions of phonological abilities to early reading acquisition. Journal of Educational Psychology 91, 450-476.

Defior, S. (2008). Jornadas de Intervencion Psicopedagogicas - $2^{\circ}$ Ciclo: Lectura y Conciencia Fonológica. Montevideo: Facultad de Psicologia. Universidad Catolica del Uruguay.

Dickinson, D. \& Newman, S. (2006). Handbook of Early Literacy Developmen, Volume 2 . New York: The Guilford Press.

Dunn, L.M., Padilla, E.R. \& Lugo, D.E. (1986). Test de Vocabulario de Imágenes Peabody. Circle Pines, MN: American Guidance Service.

Goddard, M., Durkin, K. \& Rutter, D.R. (1985). The semantic focus of maternal speech: A comment on Ninio and Bruner. Journal of Child Language, 12, 209-213.

González, M. J. (1993). Estudio evolutivo del aprendizaje de la lectura: Análisis causal de la influencia de variables de desarrollo fonológico y psicolingüístico y de variables contextuales con niños normales y con dificultades de aprendizaje, de cinco a ocho años. (Tesis doctoral) S.P.I.C.U.M. Universidad de Málaga.

Guardia, P. (2003). Relaciones entre habilidades de alfabetización emergente y la lectura desde nivel de transición mayor a primero básico. Psykhé 12, 63-79.

Herrera, L. (2002). Tesis Doctoral. Fonología y música en Educación infantil. Facultad de Ciencias de la Educación. Universidad de Granada. España.

Herrera, L., Defior, S., \& Lorenzo, O. (2007). Intervención educativa en conciencia fonológica en niños prelectores de lengua materna española y tamazight: Comparación de dos programas de entrenamiento. Infancia y aprendizaje, vol. $30 n^{\circ} 1,39-54$.

Jiménez, J., \& O'Shanahan, I. (2008). Enseñanza de la lectura: de la teoría y la investigación a la práctica educativa. Universidad de la Laguna, España. Revista Iberoamericana de Educación, 45(5). Recuperado de http://www.rieoei.org/ deloslectores/2362JimenezV2.pdf

Lesgold, A.M.;Resnick, L., \& Hammond, K. (1985). Learning to read: A longitudinal study of word skill development in two curricula. En E. Mackinnon y T. Walter (Eds.), Reading research: Advances In Theory And Practice, (Vol IV), New York: Academic Press. 
Morais, J. (1998). El arte de leer. Madrid: Visor.

Perfetti, C.A. (1986). Continuities in reading acquisition, reading skill, and reading disability. Remedial and Special Education, 7, 11-21.

Perfetti, C.A. (1992). The representation problem in reading acquisition. En P.B. Gough, L.C Ehri \& R. Treiman (Eds.) Reading acquisition (pp.145-174). Hillsdale, NJ: Lawrence Erlbaum Associates.

Piacente T, Querejeta, M, Marder, S,y Resches (2003). Evaluación del Contexto Alfabetizador. La Plata: Comisión de Investigaciones Científicas.

Piacente, T., Marder, S. Resches, M. \& Ledesma, R. (2006). El contexto alfabetizador hogareño en familias de la pobreza. Comparación de sus características con las de familias no pobres. Revista Iberoamericana de Diagnóstico y Evaluación Psicológica, 21(1), 61-88.

Querejeta, M. (2010). Sociedad, familia y aprendizaje. El papel de los contextos hogareños. Orientación y Sociedad, 10, 1-24.

Quintanal Diaz, J. (2001). Los primeros pasos en la lectura. Universidad complutense de Madrid. Madrid: C.E.S Don Bosco.

Samuelson, S., \& Lundberg, I. ( 2003). The impact of environmental factor son components of Reading and dislexia. Annals of Dyslexia 46, 201-217.

Selles Nohales, P., \& Martínez Giménez, T. (2008). Evaluación de los predictores y facilitadores de la lectura: análisis y comparación de pruebas en español y en inglés. Bordón: Revista de Orientación Pedagógica 60(3),113-129.
Serrano, F. (2005). Disléxicos en español: el papel de la fonología y la ortografía. Tesis doctoral. Universidad de Granada.

Serrano, F., \& Defior, S. (2004). Dislexia en Español: estado de la cuestión. Revista electrónica de investigación psicoeducativa y psicopedagógica 2(2), 13-34.

Stanovich, K.E. (1988). The right wrong places to look for the cognitive locus in reading disability. Annals of Dyslexia. 38, 154-177.

Whitehurst, G. \& Lonigan, Ch. (1998). Child Development and Emergent Literacy. Child Development, 69 (3), 848-872.

Wimmer,H, Mayringer ,H. \& Landerl, K. (2000). The doubledeficithypothesis and difficulties in learnin to read a regular orthography. Journal of Educational Psychology, 93(4), 668-680.

Wolf., M. \& Bowers, P. (2000) Naming-Speed Processes and Developmental Reading Disabilities: An Introduction to the Special Issue on the Double Deficit Hypothesis. Journal of Learning Disabilities. July/August, 322-324.

Wolf, M. \& Denckla, M. (2003) Rapid Automatized Naming Tests. Greeville, SC: Super Duper.

Para citar este artículo:

Cuadro, A., \& Berná, J. (2015). Inicio de la alfabetización, habilidades prelectoras y contexto alfabetizador familiar en una muestra de niños uruguayos. Ciencias Psicológicas 9(1): 7 - 14 